\title{
The deep evolutionary roots of 'addiction'
}

\author{
Moira J van Staaden ${ }^{1,2 *}$, F Scott Hall ${ }^{1,3}$ and Robert Huber ${ }^{1,2}$
}

'Juvatech creative behavioral designs, Maumee, $\mathrm{OH}$ 43537, USA

2JP Scott Center for Neuroscience, Mind \& Behavior, Department of Biological Sciences, Bowling Green State University, Bowling Green, OH 43403, USA

${ }^{3}$ Department of Pharmacology and Experimental Therapeutics, University of Toledo, Toledo, $\mathrm{OH} 43606$, USA

\section{Article Info}

\section{Article Notes}

Received: May 01, 2018

Accepted: May 24, 2018

\section{*Correspondence:}

Dr. Moira van Staaden, PhD, Bowling Green State University

JP Scott Center for Neuroscience, Mind \& Behavior

Department of Biological Sciences, Bowling Green, $\mathrm{OH}$ 43403 USA;

Telephone: +1 4193720341;

Fax: +1 4193722024;

Email: mvs.bgsu@gmail.com

C 2018 van Staaden MJ. This article is distributed under the terms of the Creative Commons Attribution 4.0 International License.

\section{Keywords}

Drug reward

Conditioned Preference

Operant Self-administration

Psychostimulant

Sensitization

Crustacea

\section{ABSTRACT}

Addiction is now recognized as a phenomenon with exceedingly deep evolutionary roots. Addictive plant alkaloids, as secondary metabolites, evolved primarily to counter insect herbivory. Although some views regard addiction as a human/mammal specific, cognitive phenomenon, we suggest that its roots are found in much more fundamental biological mechanisms for learning and motivation, mechanisms which are shared by taxa since the early evolution of bilateral metazoans. According to this view, addiction is fundamentally an invertebrate phenomenon and humans can be viewed as collateral damage in this coevolutionary arms race. Results from a variety of invertebrate preparations demonstrate behavioral and neural consequences of drug exposure, ranging from psychostimulant properties and sensitization, to conditioned cue learning and operant self-administration. This mini review focuses attention on our recent work in crayfish, but there is certainly evidence for the presence of addictionlike phenomena in more ancient invertebrate taxa.

\section{Introduction}

Addiction ${ }^{1}$ manifests in various ways, from compulsive drugseeking and consumption despite adverse consequences, perceptual changes in salience and reward strength, to reduced response inhibition $^{2,3}$. In much of the literature on addiction, aspects of the process are often viewed as distinctly mammalian, arising, in part, from the higher cognitive capacity of this taxon. Some phenomena may even be regarded as unique to primates, or even just humans. This supposition arises from the fact that studies are conducted almost exclusively in mammals, and evolutionary antecedents of the behavior have rarely been considered. Studies of drug reward and reinforcement in a more diverse range of organisms evidence that the emergence of such traits predates the emergence of a mammalian lineage by a wide margin ${ }^{4,5,6}$ (Figure 1). Taking a phylogenetic view of addiction is not entirely new. Starting as early as the mid90's Nesse (1994) proposed that an integrative evolutionary framework might result in a more nuanced understanding of addictive processes, with implications for prevention, treatment, and policy. Emerging from a genetic level ${ }^{8}$, metazoan life exhibits exceedingly deep homologies in neural mechanisms and functioning; the former observable in neural signaling ${ }^{9}$, chemical modulation ${ }^{10}$, and receptor elements ${ }^{11}$, and the latter in shared associations of neural mechanisms and behavioral contexts ${ }^{12,13}$. Here we plumb the depths of this functional homology with evidence derived from crayfish ${ }^{14}$, which combine an experimentally tractable nervous system, sophisticated behavior, 


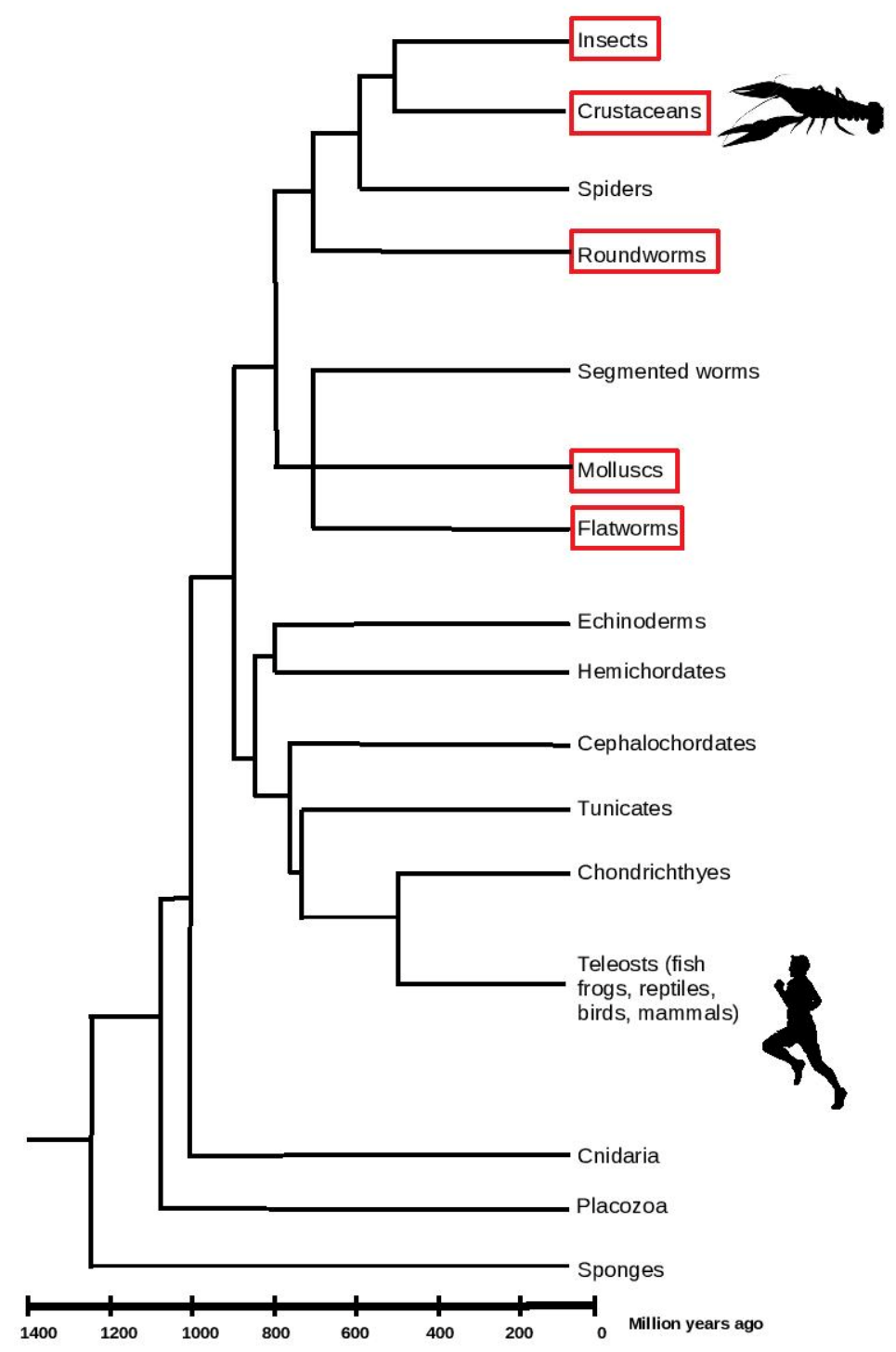

Figure 1. Phylogenetic Tree Showing the Divergence Time and Evolutionary Distance Between Phyla. Invertebrate taxa for which elements of addiction have been demonstrated are indicated in red (Insects honey bee, fruit fly, ants; Crustaceans crab, crayfish; Roundworms Caenorhabditis elegans; Molluscs snail; Flatworms planaria). Modified from Hiragaki et al., 2015.

and a demonstrated susceptibility to mammalian drugs of abuse. This system has generated insights into the full spectrum of addiction components, including the fundamental biological mechanisms of drug effects, how the seeking tendency is implemented in a 'simple' nervous system, and how motivation and reward dispositions are impacted by drugs of abuse.

The key drivers of addiction are reflected in altered (and aberrant) expressions of motivation and learning, capacities which emerged early in the precambrian ${ }^{15}$. In general, the evolutionary process has molded neural systems to maximize both of these for successful goal pursuit in an uncertain world. Exploration biases an individual's behavior to increase encounters with natural rewards and is essential for survival e.g. food, water, and other resources ${ }^{16}$. The primary drivers of these appetitive motivational states are mechanisms that increase the incentive salience of associated stimuli and prioritize encounters with novel stimuli. These natural tendencies are closely paralleled by the effects of psychostimulant drugs, particularly the capacity to activate the SEEKING drive, one of Panksepp's seven Primal Emotions ${ }^{17}$ as well as generation of rewarding signals. Invertebrate psychostimulant effects resemble those of mammals and appear to be a critical requirement for perceptions of reward in this system. All addictive drugs examined in crayfish bring about psychostimulant effects (at least at lower concentrations) as they do in mammals. This includes a wide range of substituted phenethylamines (i.e., amphetamines and cathinones ${ }^{18,19}$, alcohol ${ }^{20}$, and opioids such as morphine and heroin ${ }^{21}$. Although there appear to be differences in the effects of various drugs that may impact 
their addiction liability in mammals ${ }^{22}$ the similar locomotor activating effects of a broad range of addictive drugs in crayfish provides both a starting point for comparisons between taxa, and perhaps telling implications for how we conceptualize, research, and treat addiction.

Intoxication, Psychostimulant Properties, and Sensitization

The systemic administration of psychoactive substances generates disturbances in neural function and behaviors ranging from altered states for consciousness, cognition, perception, judgement, and affect. For instance, in a wide range of taxa, ethanol induces an initial period of disinhibition and psychomotor stimulation, followed by loss of motor and postural control ${ }^{2325}$. Crayfish are no exception. When placed in alcohol-containing water they display distinct motor deficits culminating in an inability to right themselves ${ }^{20}$. With continued exposure, individuals develop physiological tolerance to ethanol and recover much of their motor control in time ${ }^{26}$.

Many addictive, plant derived alkaloids are potent parasympathomimetics producing consistent psychoactive effects $^{27}$. For instance, although their specific biological targets and precise modes of action may vary, cocaine, nicotine, amphetamine, and cathinone all, directly or indirectly, stimulate monoamine neurotransmitter systems, in particular dopamine ${ }^{28,29}$. In crayfish, cocaine elicits rapid backwards walking and a series of defensive tail flips, before Individuals lose the ability to right themselves and assume a posture of rigid legs, abdomen, and $\operatorname{claws}^{30}$. Similar locomotor depression was report during the first of several days of repeated cocaine administration ${ }^{31}$. Subsequently, with the development of tolerance to motor impairment, the behavioral effects of cocaine turn distinctly stimulant, with increases in speed, distance traveled, and walking bouts $^{31}$. Pericardial infusions of amphetamine were excitatory over a much wider range of concentrations, with treated individuals walking along the arena's perimeter wall showing vigorous antennal movements ${ }^{30}$. In mammalian models of addiction, a cue paired with access to an addictive drug will acquire enhanced incentive salience ${ }^{32,33}$, and repeated drug exposure can impair the motivational capacity to seek natural rewards such as food and $\operatorname{sex}^{34}$. In crayfish, single doses of morphine and amphetamine induce long-term psychostimulant effects and behavioral sensitization ${ }^{18,31}$. These effects persisted even past multiday, drugfree periods or extinction trials $\mathrm{s}^{35}$. The ability of single doses of stimulant drugs to induce longterm behavioral sensitization in crayfish demonstrates the fundamental sensitivity of neural substrates to this type of chemical insult and suggests that further study might shed light on the risk factors for an individual to enter an addictive cycle ${ }^{35}$.

\section{Associative Learning}

The capacity to associate cues and consequences clearly did not arise in mammals de novo. Rather, the neural mechanisms for detecting consistency and predicting future outcomes are very deeply rooted across broad phylogenetic divisions ${ }^{36,15}$. Our understanding of the ability to associate paired events has been much enriched by the use of invertebrate preparations in both classical and operant conditioning scenarios ${ }^{37}$. With an ability to learn, animals readily associate cues and linked consequences via Pavlovian conditioning, thus imbuing cues with predictive value. Outcomes with positive valence generate appetitive responses which increase preference for, and approach to, the associated cues, whereas those perceived as aversive lead to cue avoidance and withdrawal ${ }^{38}$. The reinforcing properties of psychostimulant drugs were tested in crayfish conditioned place preference (CPP) experiments. Robust preference for the associated environment emerged when spatial conditioning paired a particular visual or tactile environment with multiday infusions of amphetamine and cocaine $^{30}$, methamphetamine ${ }^{39}$, and opioids ${ }^{35}$. Amphetamine elicited stronger drug-paired conditioning than did cocaine at comparable doses ${ }^{30}$. Although multiple instances of drug-pairing were necessary to develop the full extent of substrate conditioning, even a single drug application was sufficient to enhance cue preference.

We explored the extent to which crayfish can adjust choices in a spatially explicit paradigm, where distinct tactile and visual cues are paired with either punishment (mild electric shock) or putative reward (psychostimulant). In the former scenario, crayfish quickly learned to avoid shock-paired areas, retreating to the 'safe' substrate until the end of the trial ${ }^{40}$. Unconditioned responses can be explicitly excluded since yoked controls show no such substrate preference. In a reward scenario, entry into a specific substrate quadrant was paired with a drug bolus delivered either into the pericardial cavity or directly above the supraesophageal ganglion. Enhanced operant responding in this spatially contingent, self-administration paradigm indicates that the animals indeed learned the drug acquisition rule, and that the drug is perceived as rewarding ${ }^{41}$.

\section{Extinction, Reinstatement, and Relapse}

The emergence and relative magnitude of psychostimulant sensitization predicts the development of conditioned cue responses, e.g., in human drug craving ${ }^{42}$. Clinical findings demonstrate that learned associations between a drug and its paired cues are highly resistant to disruption through behavioral intervention ${ }^{43}$. This has focused work on mechanisms underlying extinction and reconsolidation of drug-associated cues. Addiction is widely considered to be a chronic, relapsing illness in which drug- 
seeking behavior can be reinstated by exposure to a priming dose of the addictive drug, cues present when the drugassociated behavior was formed, or exposure to physical or psychological stressors ${ }^{44}$. Experimental extinction trials present the cue but withhold the accustomed reward, such that conditioned drug seeking decreases over repeated trials, in a manner which parallels (in behavioral, neural, and conceptual terms) the extinction of conditioned fear ${ }^{45}$. An established morphine-induced CPP in crayfish was experimentally extinguished by repeated pairing of cue and saline injections. A subsequent priming dose of morphine readily reinstated the CPP in these animals following drugfree periods of up to five days ${ }^{46,35}$, demonstrating that vulnerability to relapse is similarly unrelenting in crayfish as it is in mammals ${ }^{47}$.

\section{Neural Substrates}

Our search for drug-sensitive neural substrates identified the supraesophageal ganglion, specifically the accessory lobes, as key targets for psychostimulant effects of amphetamine and cocaine ${ }^{18}$. To explore molecular responses of this region when challenged with psychostimulant drugs, we examined accessory lobe neuropil regions for molecular activation in response to cocaine reward. Enhanced cellular expression patterns of the immediate early gene $c F o s$ point to recent neural activation. The accessory lobes of cocaine-conditioned crayfish showed considerably higher cFos expression compared to saline-conditioned controls. In cocaine treated individuals, which had been placed into novel surroundings, the magnitude of $c F o s$ expression was higher still, suggesting that the effects of cocaine were amplified in the presence of novel stimuli ${ }^{31}$.

\section{Conclusions}

The parallels of mammalian and invertebrate drugassociated reward highlighted here are at the same time distinctive and somewhat surprising (absent a phylogenetic perspective). Our work on crayfish revealed a sensitivity to human drugs of abuse, and has yielded insights into a broad spectrum of addiction components. With their relatively sophisticated behavior and experimentally tractable nervous system, crayfish complement the dominant vertebrate models, effectively bridging the study of affective states and the neural substrates of addiction. Given the exceedingly diverse lifestyles and ecologies within which invertebrate reward systems operate, understanding their evolved solutions brings fresh perspective to the prevailing notion of reward systems in general. Despite considerably fewer neural elements, a distinctly segmental/modular arrangement compared to mammals, and lacking the formal structures thought essential to human addiction, invertebrate behavioral responses to drugs nonetheless bear a striking resemblance to those of amniotic vertebrates. The ability to probe reward circuits at the level of individual neurons will undoubtedly advance solutions for how deeply rooted circuits for processing and encoding rewards created systemic vulnerabilities that were exploited by plants in their efforts to deter herbivory. Can we identify the source of such vulnerability by analyzing the role of individual neurons in these circuits? Doing so will serve as a valuable hypothesis generating engine, allowing us to predict key features, and hence potential targets, for treatment of human addiction. We anticipate that studies in a wider phylogenetic range of organisms will contribute fundamental insights into neural and neurochemical mechanisms mediating responses to addictive drugs. Perhaps most crucially, identifying the limits of such comparison will determine whether there are, in fact, any distinctly mammalian or human facets to addiction arising from their higher cognitive capacity (e.g., exertion of self-control). There may well be additional functions emerging later in evolution that contribute to addictive phenomena unique to mammals. However, a long history in which such an exclusive view has gradually been eroded by the identification of 'higher' order phenomena in 'lower' taxa suggests otherwise. If human susceptibility to drugs of abuse derives from shared neural mechanisms that render us vulnerable to a coevolutionary arms race between plants and insects ( $\mathrm{see}^{27}$ for a detailed review), then it is indeed fitting that invertebrate models offer hope of accelerating the search for understanding and solutions to the problem of addiction in humans.

\section{Acknowledgements}

This work was supported in part by NSF grant DUE1525623 (MvS) and by funding from the Ohio Attorney General's Center for the Future of Forensic Science (to RH and MvS).

\section{Conflict of Interest Statement}

The authors declare that there is no conflict of interest.

\section{References}

1. American Society of Addiction Medicine (ASAM). 2011. Public Policy Statement: Definition of Addiction. www.asam.org/researchtreatment/definitionofaddiction (http://www.asam.org/researchtreatment/definitionofaddiction)

2. Koob GF. The dark side of emotion: the addiction perspective European Journal of Pharmacology. 2015; 753: 73-87.

3. Fattore L, Diana M. Drug addiction: an affectivecognitive disorder in need of a cure. Neuroscience \& Biobehavioral Reviews. 2016; 65: 341-361

4. Sovik E, Barron AB. Invertebrate models in addiction research. Brain, Behavior and Evolution 2013; 82(3):15365. doi: 10.1159/000355506

5. Barron AB, Søvik E, Cornish JL. The roles of dopamine and related compounds in rewardseeking behavior across animal phyla. Frontiers in Behavioral Neuroscience 2010; 4:163. doi: 10.3389/ fnbeh.2010.00163

6. Holland LZ. The origin and evolution of chordate nervous systems. 
Philosophical Transactions of the Royal Society B: Biological Sciences. 2015; 370(1684): 20150048. doi:10.1098/rstb.2015.0048.

7. Nesse RM. An evolutionary perspective on substance abuse. Ethology and Sociobiology. 1994; 97: 339-348.

8. Wu YC, Bansal MS, Rasmussen MD, et al. Phylogenetic Identification and Functional Characterization of Orthologs and Paralogs across Human, Mouse, Fly, and Worm. BioRxiv. 2014; 005736. doi: $10.1101 / 005736$

9. Pandey P, Mersha MD, Dhillon HS. A synergistic approach towards understanding the functional significance of dopamine receptor interactions. Journal of Molecular Signaling. 2013; 8: 13. doi:10.1186/17502187813

10. Blenau W, Thamm M. Distribution of serotonin (5HT) and its receptors in the insect brain with focus on the mushroom bodies. Lessons from Drosophila melanogaster and Apis mellifera. Arthropod Structure \& Development. 2011; 40(5): 381-394. doi:10.1016/j.asd.2011.01.004

11. Katz PS, Lillvis JL. Reconciling the deep homology of neuromodulation with the evolution of behavior. Current Opinion in Neurobiology. 2014; 29: 39-47. doi:10.1016/j.conb.2014.05.002

12. Egnor SR, Branson K. Computational Analysis of Behavior. Annual Review of Neuroscience. 2016; 39(1): 217-236. doi:10.1146/ annurevneuro070815013845

13. Kravitz EA. Serotonin and aggression: Insights gained from a lobster model system and speculations on the role of amine neurons in a complex behavior. Journal of Comparative Physiology A: Sensory, Neural, and Behavioral Physiology. 2000; 186(3): 221-238. doi: $10.1007 / \mathrm{s} 003590050423$

14. Huber R, ImehNathaniel A, Nathaniel TI, et al. Drugsensitive Reward in Crayfish: Exploring the Neural Basis of Addiction with Automated Learning Paradigms. Behavioural Processes. 2018; doi:10.1016/j. beproc.2018.03.015

15. Menzel R, Benjamin PR. Invertebrate learning and memory. Amsterdam: Elsevier Science; 2013.

16. Panksepp J, Wilson CG. Brain SEEKING Circuitry in Neuroeconomics: A Unifying Hypothesis for the Role of Dopamine-Energized Arousal of the Medial Forebrain Bundle in Enthusiasm-Guiding Decision-Making. In Neuroeconomics (pp. 231252). Berlin, Heidelberg: Springer; 2016.

17. Panksepp J. Affective neuroscience: The Foundations of Human and Animal Emotions. Oxford: Oxford University Press; 2014.

18. Alcaro A, Panksepp J, Huber R. D-amphetamine stimulates unconditioned exploration/approach behaviors in crayfish: Towards a conserved evolutionary function of ancestral drug reward Pharmacology Biochemistry and Behavior. 2011; 99(1): 7580. doi:10.1016/j.pbb.2011.04.004

19. Gore SV. Behavioral characterization of substituted amphetamines and their synthetic cathinone analogues in the Rusty crayfish (Orconectes rusticus). 2017; Unpubl. PhD dissertation. Bowling Green State University. http://rave.ohiolink.edu/etdc/view?acc num=bgsu1510511175410233

20. Swierzbinski ME, Lazarchik AR, Herberholz J. Prior social experience affects the behavioral and neural responses to acute alcohol in juvenile crayfish. Journal of Experimental Biology. 2017; 220(8): 1516-1523. doi:10.1242/jeb.154419

21. ImehNathaniel A, Okon M, Huber R, et al. Exploratory behavior and withdrawal signs in crayfish: Chronic central morphine injections and termination effects. Behavioural Brain Research. 2014; 264: 181-187.

22. Badiani A, Belin D, Epstein D, et al. Opiate versus psychostimulant addiction: the differences do matter. Nature reviews Neuroscience. 2011; 12(11): 685700. doi:10.1038/nrn3104.

23. Heinz AJ, Beck A, Meyer-Lindenberg A, et al. Cognitive and neurobiological mechanisms of alcohol-related aggression. Nature
Reviews Neuroscience. 2011; 12(7): 400-413. doi:10.1038/nrn3042

24. Moore MS, Dezazzo J, Luk AY, et al. Ethanol Intoxication in Drosophila: Genetic and Pharmacological Evidence for Regulation by the cAMP Signaling Pathway. Cell. 1998; 93(6): 997-1007. doi:10.1016/s00928674(00)812052

25. Vonghia L, Leggio L, Ferrulli A, et al. Acute alcohol intoxication. European Journal of Internal Medicine. 2008; 19(8): 561-567. doi:10.1016/j.ejim.2007.06.033

26. Strawn JR, Cooper RL. The effects of ethanol on presynaptic components of synaptic transmission in a model glutamatergic synapse: The crayfish neuromuscular junction. Comparative Biochemistry and Physiology Part C: Toxicology \& Pharmacology. 2002; 131(3): 395-404. doi:10.1016/s15320456(02)000261

27. Wink M. Plant Secondary Metabolites Modulate Insect Behavior-Steps Toward Addiction? Frontiers in Physiology Invertebrate Physiology. 2018; doi: 10.3389/fphys.2018.00364

28. Eshleman AJ, Forster MJ, Wolfrum KM, et al. Behavioral and neurochemical pharmacology of six psychoactive substituted phenethylamines: Mouse locomotion, rat drug discrimination and in vitro receptor and transporter binding and function. Psychopharmacology. 2013; 231(5): 875-888. doi:10.1007/s0021301333036

29. Pistillo F, Clementi F, Zoli M, et al. Nicotinic, glutamatergic and dopaminergic synaptic transmission and plasticity in the mesocorticolimbic system: Focus on nicotine effects. Progress in Neurobiology. 2015; 124127. doi.org/10.1016/j. pneurobio.2014.10.002.

30. Panksepp JB, Huber R. Ethological analyses of crayfish behavior: A new invertebrate system for measuring the rewarding properties of psychostimulants. Behavioural Brain Research. 2004; 153(1): 171180. doi:10.1016/j.bbr.2003.11.014

31. Nathaniel TI, Panksepp J, Huber R. Alteration of cFos mRNA in the accessory lobe of crayfish is associated with a conditionedcocaine induced reward. Neuroscience Research. 2012; 72(3): 243256. doi:10.1016/j.neures.2011.11.009

32. Berridge KC, Robinson TE, Aldridge JW. Dissecting components of reward: "liking", "wanting", and learning. Current Opinion in Pharmacology. 2009; 9(1): 65-73. doi: 10.1016/j.coph.2008.12.014.

33. Robinson MJ, Robinson TE, Berridge KC. Incentive Salience and the Transition to Addiction. Biological Research on Addiction. 2013; 2: 391-399 doi:10.1016/b9780123983350.00039x.

34. Volkow ND, Fowler JS, Wang GJ. The addicted human brain: insights from imaging studies. Journal of Clinical Investigation. 2003; 111(10): 1444-1451. doi: 10.1172/JCI200318533.

35. Nathaniel TI, Panksepp J, Huber R. Effects of a single and repeated morphine treatment on conditioned and unconditioned behavioral sensitization in Crayfish. Behavioural Brain Research. 2010; 207(2): 310-320. doi:10.1016/j.bbr.2009.10.010

36. Giurfa M, Sandoz J. Invertebrate learning and memory: Fifty years of olfactory conditioning of the proboscis extension response in honeybees. Learning \& Memory. 2012; 19(2): 5466.. doi:10.1101/ Im.024711.111.

37. Cook DG, Carew TJ. Operant conditioning of head waving in Aplysia. Proceedings of the National Academy of Sciences. 1986; 83(4): 11201124. doi:10.1073/pnas.83.4.1120.

38. Everitt BJ, Robbins TW. Drug addiction: updating actions to habits to compulsions ten years on. Annual Review of Psychology. 2016; 67: 2350.

39. ImehNathaniel A, Adedeji A, Huber R, Nathaniel TI. The rewarding properties of methamphetamine in an invertebrate model of drug addiction. Physiology \& Behavior. 2016; 153: 4046. doi:10.1016/j. physbeh.2015.10.017. 
40. Bhimani R, Huber R. Operant avoidance learning in crayfish, Orconectes rusticus: Computational ethology and the development of an automated learning paradigm. Learning \& Behavior. 2015; 44(3): 239-249. doi:10.3758/s134200150205y

41. Datta U, van Staaden M, Huber R. Crayfish selfadminister Amphetamine in a Spatially Contingent Task. Frontiers in Physiology Invertebrate Physiology. 2018; DOI: 10.3389/ fphys.2018.00433.

42. Vezina P, Leyton M. Conditioned cues and the expression of stimulant sensitization in animals and humans. Neuropharmacology. 2009; 56(Suppl 1): 160-168. doi: 10.1016/j.neuropharm.2008.06.070.

43. Taylor JR, Olausson P, Quinn JJ, Torregrossa MM. Targeting extinction and reconsolidation mechanisms to combat the impact of drug cues on addiction. Neuropharmacology. 2009; 56(Suppl 1): 186-195. Doi: 10.1016/j.neuropharm.2008.07.027

44. Kalivas PW, Volkow N, Seamans J. Unmanageable motivation in addiction: a pathology in prefront-alaccumbens glutamate transmission. Neuron. 2005; 45(5): 647-650.

45. Peters J, Kalivas PW, Quirk GJ. Extinction circuits for fear and addiction overlap in prefrontal cortex. Learning \& Memory. 2009; 16(5), 279288. doi:10.1101/lm.1041309

46. Nathaniel TI, Panksepp J, Huber R. Drugseeking behavior in an invertebrate system: Evidence of morphineinduced reward, extinction and reinstatement in crayfish. Behavioural Brain Research. 2009; 197(2), 331338. doi:10.1016/j.bbr.2008.08.043

47. Stewart J, Wise RA. Reinstatement of heroin selfadministration habits: Morphine prompts and naltrexone discourages renewed responding after extinction. Psychopharmacology. 1992; 108(12), 7984. doi:10.1007/bf02245289

48. Hiragaki S, Suzuki T, Mohamed AAM, Takeda M. Structures and functions of insect arylalkylamine Nacetyltransferase (iaaNAT); a key enzyme for physiological and behavioral switch in arthropods. Frontiers in Physiology. 2015; 6: 113. doi:10.3389/fphys.2015.00113 